\title{
TESTE DE FRIO PARA AVALIAÇÃO DO POTENCIAL FISIOLÓGICO DE SEMENTES DE PINHÃ̃ MANSO (Jatropha curcas L.)
}

\section{COLD TEST METHODOLOGY FOR EVALUATION OF THE PHYSIOLOGICAL POTENTIAL OF Jatropha curcas SEEDS}

\begin{abstract}
Glauter Lima OLIVEIRA ${ }^{1}$; Paulo Cesar HILST ${ }^{2}$; Laercio Junio da SILVA ${ }^{3}$; Marcelo Coelho SEKITA ${ }^{4}$; Denise Cunha Fernandes dos Santos DIAS ${ }^{5}$

1. Engenheiro Agrônomo, Prof $^{\circ}$ Doutor em Agronomia-Fitotecnia, Instituto Federal de Educação, Ciência e Tecnologia do Piauí IFPI, Valença do Piauí, PI, Brasil. glauteragro@ hotmail.com; 2. Engenheiro Agrônomo Doutorando em Agronomia-Fitotecnia, Universidade Federal de Viçosa - UFV, Viçosa, MG, Brasil; 3. Engenheiro Agrônomo, Prof ${ }^{\circ}$ Doutor em Agronomia-Fitotecnia, Universidade Federal de Viçosa - MG, Viçosa, MG, Brasil; 4. Engenheiro Agrônomo, Doutor em Fisiologia Vegetal, Universidade Federal de Viçosa - MG, Viçosa, MG, Brasil 5. Engenheira Agrônoma, Prof ${ }^{a}$, Doutora em Agronomia-Produção e Tecnologia de Sementes - UFV, Viçosa, MG, Brasil
\end{abstract}

\begin{abstract}
RESUMO: O pinhão manso (Jatropha curcas L.) é uma planta de grande destaque entre as oleaginosas promissoras para a produção de biodiesel. Contudo, para a comercialização segura de lotes são necessárias informações sobre a qualidade das sementes, especialmente vigor. Objetivou-se, neste trabalho, adequar a metodologia do teste de frio para a avaliação do vigor de sementes de pinhão manso. Sementes de cinco lotes de pinhão manso foram inicialmente submetidas a testes para avalição da qualidade fisiológica e, em seguida, submetidas ao teste de frio. Para isso, foram semeadas em papel toalha, tipo germitest, com e sem solo, sendo confeccionados rolos que foram mantidos a $10{ }^{\circ} \mathrm{C}$, por cinco, sete, nove e 11 dias. Após cada período, os rolos foram transferidos para germinador a $25^{\circ} \mathrm{C}$, realizando-se a avaliação da germinação aos 5 e 7 dias. O experimento foi conduzido em delineamento inteiramente casualizado e as médias dos tratamentos comparadas pelo teste de Tukey $(\mathrm{p} \leq 0,05)$ e correlacionadas com os resultados dos demais testes. $\mathrm{O}$ teste de frio é eficiente para a estratificação dos lotes de sementes de pinhão manso em níveis de vigor. Recomenda-se utilizar a metodologia do rolo de papel sem solo e manter as sementes em BOD a $10{ }^{\circ} \mathrm{C}$ por sete dias, seguida por um período de cinco dias em germinador a $25^{\circ} \mathrm{C}$.
\end{abstract}

PALAVRAS-CHAVE: Jatropha curcas L. Vigor. Teste de frio.

\section{INTRODUÇÃO}

Entre as oleaginosas prospectadas para a produção de biocombustíveis, o pinhão manso (Jatropha curcas L.) destaca-se por apresentar potencial de rendimento de óleo com alta qualidade físico-química, que pode ser usado para produção de biodiesel e bioquerosene (ARRUDA et al. 2004; DURÃES et al., 2011; FREITAS et al., 2011; LAVIOLA et al.,2012). Porém, por se tratar de uma espécie em domesticação, ainda são poucos os estudos com esta espécie, principalmente no que se refere à avaliação do potencial fisiológico de suas sementes (OLIVEIRA, 2009; PINTO et al., 2009; VANZOLINI et al., 2010).

A utilização de sementes de alta qualidade constitui-se na base para a obtenção de stands uniformes, plantas bem desenvolvidas, alta produtividade e elevado rendimento de óleo. A qualidade das sementes de maneira geral tem sido avaliada, rotineiramente, pelo teste de germinação (BRASIL, 2009), conduzido sob condições ideais, em laboratório. Desse modo, os resultados obtidos expressam o potencial máximo de germinação do lote. No entanto, estes resultados nem sempre são confirmados em campo, principalmente quando as condições de ambiente se desviam das mais adequadas. Por este motivo, atualmente, é cada vez mais crescente a procura por métodos que permitam avaliar, de maneira rápida e eficiente, o potencial fisiológico das sementes, representado pelo seu potencial de armazenamento e de emergência de plântulas em campo.

Neste contexto, o teste de frio foi inicialmente desenvolvido para avaliar a eficiência da aplicação de fungicidas às sementes, mas em decorrência do comportamento distinto de diferentes lotes submetidos ao teste, este passou a ser considerado como um teste de vigor (BARROS et al., 1999).

Assim, o teste de frio tem sido utilizado na avaliação do vigor de sementes de algumas espécies como mamona (MENDES et al., 2010), milho (CASEIRO; MARCOS FILHO, 2002), soja (SCHUAB et al 2006; VIEIRA et al., 2010), algodão (TORRES, 1998; MIGUEL et al., 2001), entre outras.

$\mathrm{O}$ teste de frio tem como principio básico a exposição das sementes a fatores adversos de baixa temperatura, alta umidade do substrato e a agentes 
patogênicos presentes no solo, quando o teste é conduzido com solo. Tais condições reduzem as chances de sobrevivência das sementes menos vigorosas, uma vez que a combinação entre baixa temperatura e alta umidade contribui para reduzir a velocidade de germinação, além de favorecer o desenvolvimento de microrganismos que podem promover a deterioração das sementes (BARROS et al., 1999). Tradicionalmente, esse teste consiste na exposição das sementes em solo não esterilizado, com teor de água ajustado para 60 ou $70 \%$ da capacidade de retenção, à temperatura de $10{ }^{\circ} \mathrm{C}$, durante sete a 10 dias, seguidos por um período de crescimento de plântulas sob temperaturas entre 25 e $30{ }^{\circ} \mathrm{C}$ (LOEFFLER et al., 1985). Contudo, estes autores constataram que o teste conduzido sem solo, ou seja, apenas em rolo de papel umedecido, apresentou sensibilidade suficiente para detectar danos causados pela secagem em sementes de milho, tendo como vantagem maior facilidade de condução e de reprodutibilidade em relação ao método com solo.

São escassos os estudos direcionados à adequação de metodologias para a avaliação do potencial fisiológico de sementes de pinhão manso. Nesse sentido, a presente pesquisa teve como objetivo adequar a metodologia do teste de frio para avaliação do potencial fisiológico de lotes de sementes de pinhão manso.

\section{MATERIAL E MÉTODOS}

A pesquisa foi conduzida no Laboratório de Sementes do Departamento de Fitotecnia da Universidade Federal de Viçosa, em Viçosa, MG. Foram utilizados cinco lotes de sementes de pinhão manso, submetidos aos seguintes testes e determinações:

\section{Germinação}

Oito subamostras de 25 sementes foram semeadas sobre duas folhas de papel toalha, tipo germitest, umedecido com quantidade de água equivalente a 2,7 vezes o peso do substrato seco. Após cobrir com mais uma folha, foram confeccionados rolos que foram mantidos em germinador a $25{ }^{\circ} \mathrm{C}$. As avaliações foram realizadas aos sete e 12 dias após a semeadura e os valores expressos em porcentagem de plântulas normais (OLIVEIRA, 2009).

\section{Primeira contagem de germinação:}

Consistiu no registro do número de plântulas normais obtidas no sétimo dia após o inicio do teste de germinação. Os valores foram expressos em porcentagem de plântulas normais.

\section{Emergência de plântulas}

Conduzido em casa de vegetação, utilizando-se bandejas de plástico contendo como substrato solo e areia (proporção de 2:1). O substrato foi umedecido inicialmente com $60 \%$ de sua capacidade de retenção máxima (BRASIL, 2009). Quatro subamostras de 50 sementes foram distribuídas em sulcos longitudinais de $2 \mathrm{~cm}$ de profundidade distanciados de $5 \mathrm{~cm}$ entre si nas bandejas de plástico. Realizaram-se, contagens diárias, registrando-se o número de plântulas emersas, ou seja, plântulas que apresentavam os cotilédones expostos acima do nível do solo, até o décimo segundo dia após a semeadura. Com os dados foram calculados o índice de velocidade de emergência (IVE) e de velocidade de emergência das plântulas (VE), segundo Nakagawa (1999), e a porcentagem final de emergência de plântulas.

\section{Comprimento de hipocótilo e radícula}

Determinado com o auxilio de régua graduada em milímetros, procedendo-se a medição do hipocótilo e da radícula das plântulas provenientes do teste de emergência. Os resultados foram expressos em cm/plântula;

\section{Massa de matéria seca de hipocótilo e radícula}

As plântulas provenientes do teste de emergência foram seccionadas em hipocótilo e radícula e colocadas para secar em estuda de circulação de ar forçada regulada na temperatura de $60{ }^{\circ} \mathrm{C}$ até atingirem peso constante. Após, realizouse a pesagem das partições (hipocótilo e radícula) em balança analítica com precisão de $0,001 \mathrm{~g}$. Os resultados foram expressos em $\mathrm{g} /$ plântula.

\section{Teste de envelhecimento acelerado}

Sementes de cada lote foram colocadas em uma camada única sobre tela metálica acoplada em caixa plástica (gerbox) contendo ao fundo $40 \mathrm{~mL}$ de água destilada. As caixas foram tampadas, de modo a se obter cerca de $100 \%$ UR em seu interior, e mantidas em câmaras tipo BOD, na temperatura de $42{ }^{\circ} \mathrm{C}$, durante 48 horas (OLIVEIRA, 2009). Após esse período, oito subamostras de 25 sementes foram submetidas ao teste de germinação. Os resultados consistiram na porcentagem de plântulas normais obtidas aos 7 dias após a semeadura.

\section{Teste de frio em rolo de papel com solo}

Oito subamostras de 25 sementes foram semeadas sobre duas folhas de papel tipo germitest umedecido com quantidade de água equivalente a 
2,7 vezes o peso do substrato seco. O papel umedecido foi mantido, previamente à montagem do teste, à temperatura de $10{ }^{\circ} \mathrm{C}$ durante 24 horas. As sementes foram distribuídas sobre duas folhas de papel e cobertas com uma fina camada de solo, proveniente de área cultivo e, a seguir, cobertas com uma terceira folha de papel, fazendo-se, então, os rolos. Esses rolos foram colocados em sacos plásticos, vedados e, em seguida, transferidos para incubadora tipo BOD à temperatura de $10{ }^{\circ} \mathrm{C}$ e, mantidos por cinco, sete, nove e 11 dias, após este períodos os mesmos foram transferidos para germinador a $25{ }^{\circ} \mathrm{C}$, realizando-se as avaliações da germinação após cinco e sete dias. A interação entre os fatores anteriormente mencionados caracterizaram os tratamentos, que encontram-se descritos na Tabela 1.

\section{Teste de frio em rolo de papel sem solo}

Foram realizados procedimentos semelhantes ao item anteriormente descrito, com exceção de que as sementes não foram cobertas por uma fina camada de solo de área de cultivo. Conforme item anterior, os tratamentos encontramse caracterizados na Tabela 1. Ao término dos testes, foi realizada a contagem do número de plântulas normais. Os resultados foram expressos em porcentagem.

Tabela 1. Descrição dos tratamentos obtidos pela interação substrato, períodos de frio e avaliação do teste de frio com sementes de pinhão manso

Legenda

RPSS $5 \mathrm{D} / 10^{\circ} \mathrm{C}-5 \mathrm{D} / 25^{\circ} \mathrm{C}$

RPSS $5 \mathrm{D} / 10^{\circ} \mathrm{C}-7 \mathrm{D} / 25^{\circ} \mathrm{C}$

RPSS $7 \mathrm{D} / 10^{\circ} \mathrm{C}-5 \mathrm{D} / 25^{\circ} \mathrm{C}$

RPSS $7 \mathrm{D} / 10^{\circ} \mathrm{C}-7 \mathrm{D} / 25^{\circ} \mathrm{C}$

RPSS 9D $/ 10^{\circ} \mathrm{C}-5 \mathrm{D} / 25^{\circ} \mathrm{C}$

RPSS 9D $/ 10^{\circ} \mathrm{C}-7 \mathrm{D} / 25^{\circ} \mathrm{C}$

RPSS $11 \mathrm{D} / 10^{\circ} \mathrm{C}-5 \mathrm{D} / 25^{\circ} \mathrm{C}$

RPSS $11 \mathrm{D} / 10^{\circ} \mathrm{C}-7 \mathrm{D} / 25^{\circ} \mathrm{C}$

RPCS 5D $/ 10^{\circ} \mathrm{C}-5 \mathrm{D} / 25^{\circ} \mathrm{C}$

RPCS $5 \mathrm{D} / 10^{\circ} \mathrm{C}-7 \mathrm{D} / 25^{\circ} \mathrm{C}$

RPCS $7 \mathrm{D} / 10^{\circ} \mathrm{C}-5 \mathrm{D} / 25^{\circ} \mathrm{C}$

RPCS $7 \mathrm{D} / 10^{\circ} \mathrm{C}-7 \mathrm{D} / 25^{\circ} \mathrm{C}$

RPCS 9D $/ 10^{\circ} \mathrm{C}-5 \mathrm{D} / 25^{\circ} \mathrm{C}$

RPCS 9D $/ 10^{\circ} \mathrm{C}-7 \mathrm{D} / 25^{\circ} \mathrm{C}$

RPCS $11 \mathrm{D} / 10^{\circ} \mathrm{C}-5 \mathrm{D} / 25^{\circ} \mathrm{C}$

RPCS $11 \mathrm{D} / 10^{\circ} \mathrm{C}-7 \mathrm{D} / 25^{\circ} \mathrm{C}$

\section{Tratamentos}

Teste de frio em rolo de papel por 5 dias $\left(10^{\circ} \mathrm{C}\right)$, com avaliação aos 5 dias após período a $25^{\circ} \mathrm{C}$ sem solo

Teste de frio em rolo de papel por 5 dias $\left(10^{\circ} \mathrm{C}\right)$, com avaliação aos 7 dias após período a $25^{\circ} \mathrm{C}$ sem solo

Teste de frio em rolo de papel por 7 dias $\left(10^{\circ} \mathrm{C}\right)$, com avaliação aos 5 dias após período a $25^{\circ} \mathrm{C}$ sem solo

Teste de frio em rolo de papel por 7 dias $\left(10^{\circ} \mathrm{C}\right)$, com avaliação aos 7 dias após período a $25^{\circ} \mathrm{C}$ sem solo

Teste de frio em rolo de papel por 9 dias $\left(10^{\circ} \mathrm{C}\right)$, com avaliação aos 5 dias após período a $25^{\circ} \mathrm{C}$ sem solo

Teste de frio em rolo de papel por 9 dias $\left(10^{\circ} \mathrm{C}\right)$, com avaliação aos 7 dias após período a $25^{\circ} \mathrm{C}$ sem solo

Teste de frio em rolo de papel por 11 dias $\left(10^{\circ} \mathrm{C}\right)$, com avaliação aos 5 dias após período a $25^{\circ} \mathrm{C}$ sem solo

Teste de frio em rolo de papel por 11 dias $\left(10^{\circ} \mathrm{C}\right)$, com avaliação aos 7 dias após período a $25^{\circ} \mathrm{C}$ sem solo

Teste de frio em rolo de papel por 5 dias $\left(10^{\circ} \mathrm{C}\right)$, com avaliação aos 5 dias após período a $25^{\circ} \mathrm{C}$ com solo

Teste de frio em rolo de papel por 5 dias $\left(10^{\circ} \mathrm{C}\right)$, com avaliação aos 7 dias após período a $25^{\circ} \mathrm{C}$ com solo

Teste de frio em rolo de papel por 7 dias $\left(10^{\circ} \mathrm{C}\right)$, com avaliação aos 5 dias após período a $25^{\circ} \mathrm{C}$ com solo

Teste de frio em rolo de papel por 7 dias $\left(10^{\circ} \mathrm{C}\right)$, com avaliação aos 7 dias após período a $25^{\circ} \mathrm{C}$ com solo

Teste de frio em rolo de papel por 9 dias $\left(10^{\circ} \mathrm{C}\right)$, com avaliação aos 5 dias após período a $25^{\circ} \mathrm{C}$ com solo

Teste de frio em rolo de papel por 9 dias $\left(10^{\circ} \mathrm{C}\right)$, com avaliação aos 7 dias após período a $25^{\circ} \mathrm{C}$ com solo

Teste de frio em rolo de papel por 11 dias $\left(10^{\circ} \mathrm{C}\right)$, com avaliação aos 5 dias após período a $25^{\circ} \mathrm{C}$ com solo

Teste de frio em rolo de papel por 11 dias $\left(10^{\circ} \mathrm{C}\right)$, com avaliação aos 7 dias após período a $25^{\circ} \mathrm{C}$ com solo
O delineamento experimental utilizado foi o inteiramente casualizado com quatro repetições. Os dados foram submetidos à análise de variância e os lotes, dentro de cada tratamento, foram comparados pelo teste Tukey $(p<0,05)$. Foram calculados ainda os coeficientes de correlação simples de Pearson (r) entre os resultados dos testes de frio e os demais testes realizados. A significância dos valores de " $r$ " 
foi determinada pelo teste " $\mathrm{t}$ " a 1 e $5 \%$ de probabilidade. A análise dos dados foi realizado com o software SAS (DELWICHE; SLAUGHTER, 2003).

\section{RESULTADOS E DISCUSSÃO}

Os resultados dos testes utilizados na caracterização dos lotes de sementes de pinhão manso (J. curcas L.) demonstraram que há diferença no potencial fisiológico das mesmas (Tabela 2). Os lotes 1, 2 e 3 apresentaram os maiores valores de germinação, porém houve diferença estatística apenas na comparação entre o lote 1 e os lotes 4 e 5 que se configuraram como os menos vigorosos. Já para a variável primeira contagem do teste de germinação, os lotes que apresentaram melhor desempenho foram o lote 1 e o lote 3 , porém apenas o lote 1 apresentou média superior às dos demais lotes (Tabela 2).

Tabela 2. Valores médios obtidos para os testes de germinação $(\mathrm{G})$; primeira contagem de germinação (PCG); emergência em solo 2:1 (Em); velocidade de emergência (VE); índice de velocidade de emergência (IVE); comprimento de hipocótilo e radícula ( $\mathrm{CH}$ e $\mathrm{CR}$ ); matéria seca de hipocótilo e radícula (MSH e MSR) e envelhecimento acelerado (EA), para cinco lotes de sementes de pinhão manso

\begin{tabular}{|c|c|c|c|c|c|}
\hline \multirow{2}{*}{ Lotes } & $\mathbf{G}$ & PCG & Em & $\mathbf{V E}$ & IVE \\
\hline & \multicolumn{3}{|c|}{ 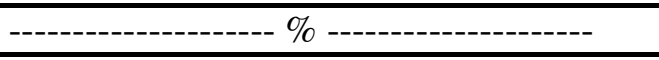 } & dias & plântulas.dia $^{-1}$ \\
\hline 1 & $89 \mathrm{a}$ & $89 \mathrm{a}$ & $89 \mathrm{a}$ & $6,82 \mathrm{a}$ & $2,94 \mathrm{a}$ \\
\hline 2 & $81 \mathrm{ab}$ & $64 \mathrm{~b}$ & $69 \mathrm{~b}$ & $6,80 \mathrm{a}$ & $2,24 \mathrm{~b}$ \\
\hline 3 & $79 a b$ & $74 \mathrm{ab}$ & $88 \mathrm{a}$ & $6,83 \mathrm{a}$ & $2,96 \mathrm{a}$ \\
\hline 4 & $78 \mathrm{~b}$ & $65 \mathrm{~b}$ & $76 \mathrm{ab}$ & $6,69 a$ & $2,52 \mathrm{ab}$ \\
\hline 5 & $78 \mathrm{~b}$ & $62 \mathrm{~b}$ & $62 \mathrm{~b}$ & $6,33 \mathrm{a}$ & $1,98 \mathrm{~b}$ \\
\hline CV $(\%)$ & 6 & 13 & 12 & 12,3 & 10,9 \\
\hline \multirow{2}{*}{ Lotes } & $\mathbf{C H}$ & CR & MSH & MSR & EA \\
\hline & \multicolumn{2}{|c|}{-------- cm.plântula ${ }^{-1}$-------- } & \multicolumn{2}{|c|}{------ g.plântula ${ }^{-1}$------ } & $---\%$--- \\
\hline 1 & $13,47 \mathrm{a}$ & $7,29 \mathrm{a}$ & $3,10 \mathrm{ab}$ & $0,55 \mathrm{ab}$ & $48 \mathrm{a}$ \\
\hline 2 & $12,83 \mathrm{ab}$ & $7,07 \mathrm{a}$ & $3,34 \mathrm{a}$ & $0,56 \mathrm{a}$ & $42 \mathrm{ab}$ \\
\hline 3 & $13,11 \mathrm{ab}$ & $7,07 \mathrm{a}$ & $3,02 \mathrm{~b}$ & $0,54 \mathrm{ab}$ & $31 \mathrm{ab}$ \\
\hline 4 & $12,34 \mathrm{~b}$ & $7,08 \mathrm{a}$ & $3,04 \mathrm{~b}$ & $0,53 \mathrm{ab}$ & $37 \mathrm{ab}$ \\
\hline 5 & $11,22 \mathrm{c}$ & $6,56 \mathrm{a}$ & $2,31 \mathrm{c}$ & $0,43 \mathrm{~b}$ & $23 \mathrm{~b}$ \\
\hline $\mathrm{CV}(\%)$ & 3,7 & 6,3 & 4,3 & 12 & 25,7 \\
\hline
\end{tabular}

Médias seguidas pela mesma letra na coluna não diferem estatisticamente entre si pelo teste de Tukey $(\mathrm{p} \leq 0,05)$.

Quanto à percentagem final de emergência de plântulas em solo, verificou-se que o melhor desempenho foi obtido pelos lotes 1,3 e 4 , resultado este que também foi observado para o número de plântulas germinadas por dia (IVE), em que os lotes 1,3 e 4 apresentaram maior potencial fisiológico ao se comparar com os demais lotes (2 e 5) (Tabela 2). Já o teste de velocidade de emergência (VE), que indica o número de dias necessários para o estabelecimento da germinação das sementes em condições de campo, não permitiu detectar diferenças no vigor dos lotes testados.

Pode-se ainda observar na Tabela 2 que os lotes que apresentaram os maiores índices de germinação (lotes 1, 2 e 3) foram os mesmos para os quais se os maiores incrementos em comprimento da parte aérea (comprimento de hipocótilo), fato este parcialmente corroborado pelo ganho de biomassa seca observado com a pesagem do hipocótilo das plântulas, sendo que os lotes 1 e 2 foram os que apresentaram um maior acumulo de biomassa vegetal. Entretanto, não se observou variações estatísticas quanto ao comprimento de raízes das plântulas oriundas dos diferentes lotes (Tabela 2), enquanto que para a biomassa seca de raiz verificou-se diferença estatística apenas entre os lotes 2 e 5 (Tabela 2).

No teste de envelhecimento acelerado, houve diferença estatística apenas entre os lotes $1 \mathrm{e}$ 5. Porém, a germinação dos lotes após o envelhecimento foi drasticamente reduzida, mesmo assim o teste mostrou-se eficiente em estratificar os 
mesmos, como visto também por Oliveira (2009) em estudo de adequação de protocolo para a condução do teste de envelhecimento acelerado para a cultura do pinhão manso.

No geral, comparando-se os testes utilizados para caracterização fisiológica dos lotes (Tabela 2), é possível observa que existe diferença na qualidade fisiológica entre os lotes e que é possível classificálos em níveis de vigor. Dessa maneira os lotes 1 e 3 podem ser classificados como os mais vigorosos, enquanto que os lotes 2 e 4 encontram-se em um estagio intermediário de vigor e o lote 5 como o de baixo vigor.

Pelo teste de frio (Tabela 3), verifica-se que, em todos os tratamentos utilizados foi possível verificar a separação dos lotes em diferentes níveis de vigor à semelhança da emergência em campo. Porém, os mais eficientes foram os tratamentos que utilizaram rolo de papel sem solo como substrato, resfriados por sete e 11 dias, tendo como períodos de avaliações finais aos cinco e sete dias de exposição à temperatura de $25^{\circ} \mathrm{C}$ (RPSS $7 \mathrm{D} / 10^{\circ} \mathrm{C}$ $5 \mathrm{D} / 25^{\circ} \mathrm{C}$ e $\left.\operatorname{RPSS} 11 \mathrm{D} / 10^{\circ} \mathrm{C}-7 \mathrm{D} / 25^{\circ} \mathrm{C}\right)$; e o teste de frio conduzido em rolo de papel com solo resfriado por 11 dias, tendo como período de avaliação final sete dias (RPCS $11 \mathrm{D} / 10^{\circ} \mathrm{C}-7 \mathrm{D} / 25^{\circ} \mathrm{C}$ ), nos quais houve separação dos lotes em três níveis de vigor. No entanto, o teste de frio conduzido em rolo de papel com solo por 5 dias $\left(10^{\circ} \mathrm{C}\right)$, com avaliação final aos 5 dias após período a $25{ }^{\circ} \mathrm{C}$ (RPCS $\left.5 \mathrm{D} / 10^{\circ} \mathrm{C}-5 \mathrm{D} / 25^{\circ} \mathrm{C}\right)$, também demonstrou resultados promissores quanto a separação dos lotes em níveis de vigor, pois o mesmo estratificou os cinco lotes em quatro níveis de vigor. Esses resultados se assemelham aos obtidos por Miguel et al. (2001), que ao trabalharem com diferentes procedimentos para a condução do teste de frio em sementes de algodão verificaram que este teste quando é realizado em rolo de papel é mais eficiente para estratificar os lotes de sementes de algodão em níveis de vigor quando comparados com o método tradicional (caixas plásticas contendo solo resfriado a $10{ }^{\circ} \mathrm{C}$ e umedecido com $70 \%$ de sua capacidade de retenção). Esses mesmos autores obtiveram resultados menos drásticos que o procedimento tradicional quando o teste de frio foi conduzido em rolo de papel com e sem solo. Kappes, Carvalho e Yamashita (2009) e Mendes et al. (2010), também obtiveram sucesso na classificação de lotes de sementes de soja e mamona, respectivamente, em níveis de vigor ao utilizarem o teste de frio em rolo de papel sem solo a $10{ }^{\circ} \mathrm{C}$ por sete dias com avaliação final aos cinco dias após exposição a 25 ${ }^{\circ} \mathrm{C}$ para a retomada do processo germinativo e do desenvolvimento das plântulas.

De acordo com Caseiro e Marcos Filho (2002), o teste de frio quando conduzido pela metodologia tradicional causa maior desuniformidade na porcentagem de plântulas normais, e como conseqüência torna o teste menos preciso e eficiente.

Tabela 3. Valores médios de plântulas normais (\%) obtidas para os cinco lotes de sementes de pinhão manso em função dos tratamentos do teste de frio

\begin{tabular}{|c|c|c|c|c|c|c|}
\hline \multirow{3}{*}{ Tratamentos } & \multicolumn{5}{|c|}{ Lotes } & \multirow{3}{*}{$\mathrm{CV}(\%)$} \\
\hline & 1 & 2 & 3 & 4 & 5 & \\
\hline & \multicolumn{5}{|c|}{------------------------- \% ------------------------- } & \\
\hline RPSS $5 \mathrm{D} / 10^{\circ} \mathrm{C}-5 \mathrm{D} / 25^{\circ} \mathrm{C}$ & $91 \mathrm{a}$ & $76 \mathrm{a}$ & $89 \mathrm{a}$ & $85 \mathrm{a}$ & $16 \mathrm{~b}$ & 10,9 \\
\hline RPSS $5 \mathrm{D} / 10^{\circ} \mathrm{C}-7 \mathrm{D} / 25^{\circ} \mathrm{C}$ & $97 \mathrm{a}$ & $83 \mathrm{a}$ & $94 \mathrm{a}$ & 89 a & $24 \mathrm{~b}$ & 9,7 \\
\hline RPSS $7 \mathrm{D} / 10^{\circ} \mathrm{C}-5 \mathrm{D} / 25^{\circ} \mathrm{C}$ & $90 \mathrm{a}$ & $70 \mathrm{~b}$ & $94 \mathrm{a}$ & $85 \mathrm{ab}$ & $24 \mathrm{c}$ & 9,6 \\
\hline RPSS $7 \mathrm{D} / 10^{\circ} \mathrm{C}-7 \mathrm{D} / 25^{\circ} \mathrm{C}$ & $91 \mathrm{a}$ & $71 \mathrm{~b}$ & 94 a & $86 \mathrm{a}$ & $23 \mathrm{c}$ & 9 \\
\hline RPSS 9D $/ 10^{\circ} \mathrm{C}-5 \mathrm{D} / 25^{\circ} \mathrm{C}$ & $95 \mathrm{a}$ & $70 \mathrm{~b}$ & $88 \mathrm{ab}$ & $80 \mathrm{ab}$ & $27 \mathrm{c}$ & 11,9 \\
\hline RPSS 9D/ $10^{\circ} \mathrm{C}-7 \mathrm{D} / 25^{\circ} \mathrm{C}$ & $97 \mathrm{a}$ & $76 \mathrm{~b}$ & $94 \mathrm{ab}$ & $87 \mathrm{ab}$ & $24 \mathrm{c}$ & 11,5 \\
\hline RPSS $11 \mathrm{D} / 10^{\circ} \mathrm{C}-5 \mathrm{D} / 25^{\circ} \mathrm{C}$ & $97 \mathrm{a}$ & $80 \mathrm{~b}$ & $96 \mathrm{a}$ & $92 \mathrm{ab}$ & $35 \mathrm{c}$ & 7,7 \\
\hline RPSS $11 \mathrm{D} / 10^{\circ} \mathrm{C}-7 \mathrm{D} / 25^{\circ} \mathrm{C}$ & $98 \mathrm{a}$ & $81 \mathrm{~b}$ & $98 \mathrm{a}$ & $93 \mathrm{a}$ & $43 \mathrm{c}$ & 4,7 \\
\hline RPCS $5 \mathrm{D} / 10^{\circ} \mathrm{C}-5 \mathrm{D} / 25^{\circ} \mathrm{C}$ & $94 \mathrm{ab}$ & $79 c$ & $97 \mathrm{a}$ & $87 \mathrm{bc}$ & $28 \mathrm{~d}$ & 5,6 \\
\hline $\mathrm{RPCS} 5 \mathrm{D} / 10^{\circ} \mathrm{C}-7 \mathrm{D} / 25^{\circ} \mathrm{C}$ & $95 \mathrm{a}$ & $91 \mathrm{a}$ & $97 \mathrm{a}$ & $92 \mathrm{a}$ & $34 \mathrm{~b}$ & 6,6 \\
\hline RPCS $7 D / 10^{\circ} \mathrm{C}-5 \mathrm{D} / 25^{\circ} \mathrm{C}$ & $83 \mathrm{a}$ & $84 \mathrm{a}$ & $88 \mathrm{a}$ & $92 \mathrm{a}$ & $35 \mathrm{~b}$ & 11 \\
\hline RPCS $7 \mathrm{D} / 10^{\circ} \mathrm{C}-7 \mathrm{D} / 25^{\circ} \mathrm{C}$ & $92 \mathrm{a}$ & $87 \mathrm{a}$ & $89 a$ & 89 a & $39 \mathrm{~b}$ & 11 \\
\hline $\mathrm{RPCS} 9 \mathrm{D} / 10^{\circ} \mathrm{C}-5 \mathrm{D} / 25^{\circ} \mathrm{C}$ & $93 \mathrm{a}$ & $87 \mathrm{a}$ & $94 \mathrm{a}$ & $88 \mathrm{a}$ & $31 \mathrm{~b}$ & 8,8 \\
\hline $\mathrm{RPCS} 9 \mathrm{D} / 10^{\circ} \mathrm{C}-7 \mathrm{D} / 25^{\circ} \mathrm{C}$ & 98 a & $87 \mathrm{a}$ & $94 \mathrm{a}$ & $88 \mathrm{a}$ & $32 \mathrm{~b}$ & 8,7 \\
\hline $\mathrm{RPCS} 11 \mathrm{D} / 10^{\circ} \mathrm{C}-5 \mathrm{D} / 25^{\circ} \mathrm{C}$ & $93 \mathrm{a}$ & $78 \mathrm{a}$ & $93 \mathrm{a}$ & $86 \mathrm{a}$ & $34 \mathrm{~b}$ & 9,7 \\
\hline RPCS $11 \mathrm{D} / 10^{\circ} \mathrm{C}-7 \mathrm{D} / 25^{\circ} \mathrm{C}$ & $96 \mathrm{a}$ & $79 \mathrm{~b}$ & $95 \mathrm{ab}$ & $91 \mathrm{ab}$ & $42 \mathrm{c}$ & 9,2 \\
\hline
\end{tabular}

Médias seguidas pela mesma letra na linha não diferem estatisticamente entre si pelo teste de Tukey ( $\mathrm{p} \leq 0,05)$. 
Os procedimentos que não utilizaram solo apresentaram resultados mais severos para o numero de plântulas normais (Tabela 3), permitindo uma melhor classificação dos lotes de sementes de pinhão manso em níveis de vigor. Também pôde-se observar que o tempo de exposição ao frio não foi um fator limitante à condução do teste de frio realizado sem solo, ou seja, mesmo nos períodos menos intensos de frio foi possível estratificar os lotes de sementes em níveis de vigor, a exemplo dos testes de frio conduzidos em rolo de papel sem solo por 7 dias $\left(10^{\circ} \mathrm{C}\right)$, com avaliação aos 5 e 7 dias após período a $25{ }^{\circ} \mathrm{C}$ (RPSS $7 \mathrm{D} / 10^{\circ} \mathrm{C}-5 \mathrm{D} / 25^{\circ} \mathrm{C}$ e RPSS $\left.7 \mathrm{D} / 10^{\circ} \mathrm{C}-7 \mathrm{D} / 25^{\circ} \mathrm{C}\right)$. Resultados semelhantes foram observados por Guiscem et al. (2010), ao estudarem o comportamento de sementes de feijão frade (Vigna unguiculata (L.) Walp) ao teste de frio em rolo de papel sem solo em diversas variações de temperatura e períodos de exposição à baixa temperatura. Diferentemente do que foi previsto por Bruggink et al. (1991), Kikuti, Pinho e Resende (1999) e Miguel et al., (2001), que verificaram que o aumento do período de exposição ao frio traz como consequiência a redução do potencial fisiológico de sementes de milho e algodão.

Quando observamos apenas os tratamentos que utilizaram solo para a condução do teste de frio (Tabela 3), podemos afirma que o aumento do período de exposição ao frio ocasionou uma redução no surgimento de plântulas normais, fato este provavelmente ocasionado pela interação entre $o$ período de exposição ao frio, a umidade e o potencial patogênico dos microrganismos encontrados nesse solo. Resultados semelhantes foram observados por Kikuti, Pinho e Resende (1999) ao trabalharem com sementes de milho, estes autores afirmam que o prolongamento do período de exposição das sementes ao frio foi um dos fatores essenciais para a redução do porcentual de plântulas normais.
Dos oito tratamentos que se mostraram eficientes na classificação dos lotes em níveis de vigor, em apenas dois tratamentos dos que utilizaram a adição do solo como fator de avaliação do teste (RPCS $5 \mathrm{D} / 10^{\circ} \mathrm{C}-5 \mathrm{D} / 25^{\circ}$ e RPCS $11 \mathrm{D} / 10^{\circ} \mathrm{C}$ $7 \mathrm{D} / 25^{\circ} \mathrm{C}$ ) foram eficientes. No teste de frio, a utilização de solo proveniente de área de cultivo tem a função de expor as sementes à agentes patogênicos (BARROS et al., 1999), enquanto que o teste de frio conduzido sem a adição de solo, avalia diretamente os efeitos da baixa temperatura e da alta umidade sobre o processo de germinação das sementes e do desenvolvimento das plântulas, sem a interferência de outros fatores bióticos (LOEFFLER et al., 1985). Em sementes de algodão, o teste de frio realizado em rolo de papel sem solo também foi o mais indicado para avaliar o vigor das sementes (MIGUEL et al., 2001), assim como em sementes de feijão o uso do rolo de papel sem terra mostrou maiores correlações com os outros testes de vigor, devido a menor variabilidade de resultados (MIGUEL; CICERO, 1999).

Comparando-se os coeficientes de correlação de Pearson entre os tratamentos do teste de frio e os demais testes aplicados às sementes (Tabela 4), observou-se que nenhum dos tratamentos testados correlacionou-se com o teste de germinação e o de primeira contagem de germinação. Porém os tratamentos para a condução do teste de frio em rolo de papel sem solo por 7 dias $\left(10^{\circ} \mathrm{C}\right)$, com avaliações aos 5 e 7 dias (RPSS $7 \mathrm{D} / 10^{\circ} \mathrm{C}-5 \mathrm{D} / 25^{\circ} \mathrm{C}$ e RPSS $7 \mathrm{D} / 10^{\circ} \mathrm{C}-7 \mathrm{D} / 25^{\circ} \mathrm{C}$ ); teste de frio em rolo de papel sem solo por 9 e 11 dias $\left(10^{\circ} \mathrm{C}\right)$, com avaliações aos 5 e 7 dias respectivamente (RPSS $9 D / 10^{\circ} \mathrm{C}-5 \mathrm{D} / 25^{\circ} \mathrm{C}$ e RPSS $\left.11 \mathrm{D} / 10^{\circ} \mathrm{C}-7 \mathrm{D} / 25^{\circ} \mathrm{C}\right)$ e o teste de frio em rolo de papel com solo por 11 dias $\left(10^{\circ} \mathrm{C}\right)$, com avaliações aos 7 dias (RPCS $11 \mathrm{D} / 10^{\circ} \mathrm{C}-7 \mathrm{D} / 25^{\circ} \mathrm{C}$ ), apresentaram alto valor de correlação com o teste de emergência.

Tabela 4. Coeficientes de correlação de Pearson entre os dados obtidos no teste de frio com sementes de pinhão manso e os demais testes: germinação $(\mathrm{G})$; primeira contagem de germinação (PCG); emergência em solo (Em); velocidade de emergência (VE); índice de velocidade de emergência (IVE); comprimento de hipocótilo e radícula (CH e CR); massa seca de hipocótilo, radícula (MSH, MSR) e envelhecimento acelerado (EA)

\begin{tabular}{ccccccccccc}
\hline \multirow{2}{*}{ Tratamentos } & \multicolumn{10}{c}{ Testes de Vigor } \\
\cline { 2 - 11 } & G & PCG & Em & VE & IVE & CH & CR & MSH & MSR & EA \\
\hline RPSS 5D $/ 10^{\circ} \mathrm{C}-5 \mathrm{D} / 25^{\circ} \mathrm{C}$ & 0,42 & 0,56 & 0,82 & $0,96 *$ & 0,82 & $0,91 *$ & $0,96 * *$ & 0,88 & $0,94 *$ & 0,74 \\
RPSS 5D $/ 10^{\circ} \mathrm{C}-7 \mathrm{D} / 25^{\circ} \mathrm{C}$ & 0,44 & 0,57 & 0,82 & $0,97 * *$ & 0,82 & $0,92 *$ & $0,96 * *$ & $0,89 *$ & $0,95 *$ & 0,76 \\
RPSS 7D $/ 10^{\circ} \mathrm{C}-5 \mathrm{D} / 25^{\circ} \mathrm{C}$ & 0,39 & 0,59 & $0,88 *$ & $0,93 *$ & $0,89 *$ & $0,89 *$ & $0,93 *$ & 0,80 & $0,89 *$ & 0,66 \\
RPSS 7D $/ 10^{\circ} \mathrm{C}-7 \mathrm{D} / 25^{\circ} \mathrm{C}$ & 0,39 & 0,59 & $0,87 *$ & $0,93 *$ & $0,88 *$ & $0,90 *$ & $0,93 *$ & 0,81 & $0,89 *$ & 0,67 \\
RPSS 9D $/ 10^{\circ} \mathrm{C}-5 \mathrm{D} / 25^{\circ} \mathrm{C}$ & 0,53 & 0,70 & $0,90^{*}$ & $0,94 *$ & $0,90 *$ & $0,94 *$ & $0,97 * *$ & 0,80 & $0,90 *$ & 0,75 \\
RPSS 9D $110^{\circ} \mathrm{C}-7 \mathrm{D} / 25^{\circ} \mathrm{C}$ & 0,47 & 0,62 & 0,85 & $0,96 * *$ & 0,86 & $0,93 *$ & $0,97 * *$ & 0,86 & $0,93 *$ & 0,75
\end{tabular}


Teste de frio para avaliação...

OLIVEIRA, G. L. et al

\begin{tabular}{lcccccccccc} 
RPSS $11 \mathrm{D} / 10^{\circ} \mathrm{C}-5 \mathrm{D} / 25^{\circ} \mathrm{C}$ & 0,42 & 0,59 & 0,85 & $0,94 *$ & 0,86 & $0,90 *$ & $0,95 *$ & 0,83 & $0,91 *$ & 0,71 \\
RPSS $11 \mathrm{D} / 10^{\circ} \mathrm{C}-7 \mathrm{D} / 25^{\circ} \mathrm{C}$ & 0,42 & 0,61 & $0,87 *$ & $0,93 *$ & $0,88 *$ & $0,90 *$ & $0,94 *$ & 0,81 & $0,90 *$ & 0,69 \\
RPCS 5D $/ 10^{\circ} \mathrm{C}-5 \mathrm{D} / 25^{\circ} \mathrm{C}$ & 0,41 & 0,58 & 0,86 & $0,96 *$ & 0,86 & $0,92 *$ & $0,94 *$ & 0,85 & $0,92 *$ & 0,70 \\
RPCS 5D $/ 10^{\circ} \mathrm{C}-7 \mathrm{D} / 25^{\circ} \mathrm{C}$ & 0,38 & 0,49 & 0,76 & $0,97 * *$ & 0,77 & $0,90 *$ & $0,94 *$ & $0,92 *$ & $0,97 * *$ & 0,74 \\
RPCS 7D $/ 10^{\circ} \mathrm{C}-5 \mathrm{D} / 25^{\circ} \mathrm{C}$ & 0,25 & 0,36 & 0,69 & $0,92 *$ & 0,70 & 0,81 & $0,90 *$ & $0,90 *$ & $0,94 *$ & 0,68 \\
RPCS 7D $/ 10^{\circ} \mathrm{C}-7 \mathrm{D} / 25^{\circ} \mathrm{C}$ & 0,42 & 0,50 & 0,75 & $0,97 * *$ & 0,75 & $0,90 *$ & $0,96 * *$ & $0,93 *$ & $0,97 * *$ & 0,78 \\
RPCS 9D $/ 10^{\circ} \mathrm{C}-5 \mathrm{D} / 25^{\circ} \mathrm{C}$ & 0,40 & 0,52 & 0,78 & $0,98 *$ & 0,79 & $0,92 *$ & $0,95 *$ & $0,91 *$ & $0,97 * *$ & 0,75 \\
RPCS 9D $/ 10^{\circ} \mathrm{C}-7 \mathrm{D} / 25^{\circ} \mathrm{C}$ & 0,47 & 0,58 & 0,81 & $0,98 * *$ & 0,81 & $0,94 *$ & $0,97 * *$ & $0,90 *$ & $0,96 * *$ & 0,78 \\
RPCS $11 \mathrm{D} / 10^{\circ} \mathrm{C}-5 \mathrm{D} / 25^{\circ} \mathrm{C}$ & 0,43 & 0,60 & 0,86 & $0,95 *$ & 0,86 & $0,92 *$ & $0,95 *$ & 0,84 & $0,92 *$ & 0,72 \\
RPCS $11 \mathrm{D} / 10^{\circ} \mathrm{C}-7 \mathrm{D} / 25^{\circ} \mathrm{C}$ & 0,43 & 0,61 & $0,87 *$ & $0,93 *$ & 0,88 & $0,90 *$ & $0,95 *$ & 0,81 & $0,90 *$ & 0,70 \\
\hline
\end{tabular}

**Significativo a $1 \%$ de probabilidade; *Significativo a $5 \%$ de probabilidade

Na Tabela 4 são apresentados dos dados de correlação entre os tratamentos e os demais testes de vigor. Observa-se que a maioria dos tratamentos apresentou correlação significativa com as demais avaliações. Nenhum tratamento apresentou correlação significativa com o teste de envelhecimento acelerado. Nesse teste, as sementes são submetidas a condições de alta temperatura e umidade relativa, podendo-se estimar a sua longevidade (OLIVEIRA, 2009; PEREIRA et al., 2012). Ao contrário, o teste de frio avalia a tolerância das sementes à baixa temperatura, sendo que o lote que melhor resistir a essa condição apresenta capacidade para germinar sob ampla variação das condições de umidade e temperatura do solo (CICERO; VIEIRA, 1994).

Entre os tratamentos testados, foi possível destacar os tratamentos para a condução do teste de frio em rolo de papel sem solo por 7 dias $\left(10^{\circ} \mathrm{C}\right)$, com avaliações aos 5 e 7 dias (RPSS $7 \mathrm{D} / 10^{\circ} \mathrm{C}$ $5 \mathrm{D} / 25^{\circ} \mathrm{C}$ e RPSS $\left.7 \mathrm{D} / 10^{\circ} \mathrm{C}-7 \mathrm{D} / 25^{\circ} \mathrm{C}\right)$; teste de frio em rolo de papel sem solo por 9 e 11 dias $\left(10^{\circ} \mathrm{C}\right)$, com avaliações aos 5 e 7 dias (RPSS 9D/ $10^{\circ} \mathrm{C}$ $5 \mathrm{D} / 25^{\circ} \mathrm{C}$ e RPSS $\left.11 \mathrm{D} / 10^{\circ} \mathrm{C}-7 \mathrm{D} / 25^{\circ} \mathrm{C}\right)$ e o teste de frio em rolo de papel com solo por 11 dias $\left(10^{\circ} \mathrm{C}\right)$, com avaliações aos 7 dias (RPCS $11 \mathrm{D} / 10^{\circ} \mathrm{C}$ $7 \mathrm{D} / 25^{\circ} \mathrm{C}$ ) como os que apresentaram correlação significativa com o teste de emergência e com a maioria dos testes de vigor aplicados às sementes de pinhão manso (Tabela 4). Esses tratamentos classificaram os lotes de sementes de pinhão manso em três níveis de vigor, quando comparado a germinação obtida após a realização do teste (Tabela 3).
Desta forma, ao levarmos em consideração o menor tempo necessário para execução do teste, a maior praticidade e a menor variação dos dados obtidos, podemos indicar como sendo o procedimento mais adequado para a condução do teste de frio para à avaliação do vigor de sementes de pinhão manso a combinação rolo de papel sem solo por sete dias $\left(10{ }^{\circ} \mathrm{C}\right)$ com avaliação da germinação cinco dias após a exposição à temperatura de $25{ }^{\circ} \mathrm{C}$ (RPSS $7 \mathrm{D} / 10^{\circ} \mathrm{C}-5 \mathrm{D} / 25^{\circ} \mathrm{C}$ ). Esse tratamento foi eficiente em classificar os lotes em três níveis de vigor, identificou os lotes 1 e 3 como os mais vigorosos e o lote 5 como o menos vigoroso, conforme apresentado na Tabela 1, e entre os tratamentos que se destacaram é o que demanda menor tempo para sua execução (12 dias).

\section{CONCLUSÃO}

O teste de frio é eficiente na classificação de lotes de pinhão manso em níveis de vigor. Assim, para a condução do teste, deve ser utilizado como substrato papel toalha, tipo germitest, sem uso de solo, e as sementes devem ser mantidas em BOD com temperatura ajustada para $10^{\circ} \mathrm{C}$ por 7 dias e em seguida transferidas para germinador com temperatura ajustada para $25^{\circ} \mathrm{C}$ por 5 dias.

\section{AGRADECIMENTOS}

Os autores agradecem ao $\mathrm{CNPq}$ e à FAPEMIG pelo apoio financeiro concedido e pela concessão da bolsa de doutorado ao primeiro autor.

\footnotetext{
ABSTRACT: The physic nut has great potential for biodiesel production. However, for safe comercialization of the lots, information about seed quality is necessary, especially about vigor. This work aimed to adapt the cold test methodology for evaluation of the physiological quality of Jatropha curcas seeds. Seeds of five lots were initially characterized and then tested with cold test. For this, seeds were sown on germitest paper, with and without soil, and rollers were made. Then, the seeds were exposed to $10{ }^{\circ} \mathrm{C}$ during five, seven, nine and 11 days. After the cold period, the seed were transferred to germinator with temperature adjusted to $25^{\circ} \mathrm{C}$ for later evaluation at 5 and 7 days. The statistical
} 
design was completely randomized and the treatment means were compared by Tukey test $(\mathrm{p} \leq 0,05)$ and correlated with the results of other tests. The cold test proved to be efficient for the lots stratification on levels of vigor. It is recommended to use paper roll without soil and keep the seeds in BOD at $10{ }^{\circ} \mathrm{C}$ for seven days and then transfer them to germinator with temperature adjusted to $25{ }^{\circ} \mathrm{C}$ for five days.

KEYWORDS: Jatropha curcas L.. Vigor. Seed analysis.

\section{REFERÊNCIAS}

ARRUDA, F. P.; BELTRÃO, N. E. M.; ANDRADE, A. P.; PEREIRA, W. E.; SEVERINO, L. S. Cultivo de pinhão manso (Jatropha curcas L.) como alternativa para o semi-árido nordestino. Revista Brasileira de Oleaginosas e Fibrosas, Campina Grande, v. 8, n. 1, p. 789-799, 2004.

BARROS, S. R. B.; DIAS, M. C. L. L.; CICERO, S. M.; KRZYZANOWSKI, F. C. Teste de frio. In: KRZYZANOWSKI, F. C.; VIEIRA, R. D.; FRANÇA NETO, J. B. (Ed.). Vigor de sementes: conceitos e testes. Londrina: ABRATES, 1999. Cap 5, p. 1-15.

BRASIL. Ministério da Agricultura e Reforma Agrária. Regras para análise de sementes. Brasília: SNDA/DNDV/CLAV, 2009. 365p.

BRUGGINK, H.; KRAAK, H. L.; BEKENDAM, J. Some factors affecting maize (Zea mays L.) cold test results. Seed Science and Technology, Zürich, v. 19, n. 1, p. 15-23, 1991.

CASEIRO, R. F.; MARCOS FILHO, J. Procedimentos para condução do teste de frio em sementes de milho: pré resfriamento e distribuição do substrato no interior da câmara fria. Revista Brasileira de Sementes, Londrina, v. 24, n. 2, p. 6-11, 2002.

CÍCERO, S. M.; VIEIRA, R. D. Teste de frio. In: VIEIRA, R. D.; CARVALHO, N. M. (Ed.). Testes de vigor em sementes. Jaboticabal: FUNEP, 1994. p. 151-164.

DELWICHE, L. D.; SLAUGHTER, S. J. The Little SAS Book: A Primer. Cary: SAS Institute, 2003. 268p.

DURÃES , F. O. M.; LAVIOLA , B. G.; ALVES, A. A. Potential and challenges in making physic nut (Jatropha curcas L.) a viable biofuel crop: the Brazilian perspective. CAB Reviews: Perspectives in Agriculture, Veterinary Science, Nutrition and Natural Resources, v. 6, n. 1, p. 1-8, 2011.

FREITAS, R. G.; MISSIO, R. F.; MATOS, F. S.; RESENDE, M. D. V.; DIAS, L. A. S. Genetic evaluation of Jatropha curcas: an important oilseed for biodiesel production. Genetics and Molecular Research, v. 10, n. 1, p. 1490-1498, 2011. http://dx.doi.org/10.4238/vol10-3gmr1146

GUISCEM, J. M.; FARIAS, A. S.; FIGUEIREDO, R. T.; CHAVES, A. M. S.; FIGUEIREDO, B. T.; PEREIRA, C. F.; ARÁUJO, R. G.; MARTINS, M. R.. Teste de frio e envelhecimento acelerado na avaliação de vigor de sementes de feijão-frade. Revista de Ciências Agrárias, Lisboa, v. 33, n. 1, p. 182-191, 2010.

KAPPES, C.; CARVALHO, M. A. C.; YAMASHITA, O. M. Potencial fisiologico de sementes de soja dessecadas com diquat e paraquat. Scientia Agraria, Piracicaba, v. 10, n. 1, p. 001-006, 2009.

KIKUTI, A. L. P.; PINHO, E. V. R. V.; REZENDE, M. L. Estudos de metodologias para a condução do teste de frio em sementes de milho. Revista Brasileira de Sementes, Londrina, v. 21, n. 2, p. 175-179, 1999.

LAVIOLA , B. G.; ALVES , A. A.; GURGEL , F. DE L.; ROSADO, T. B.; COSTA, R. D.; ROCHA , R. B. Estimate of genetic parameters and predicted gains with early selection of physic nut families. Ciência e Agrotecnologia, Lavras, v. 36, n. 1, p. 163-170, 2012. 
LOEFFLER, T. M.; MEYER, J. L.; BURRIS, J. S. Comparison of two test procedures for use in maize drying studies. Seed Science and Technology, Zürich, v. 13, p. 653-658, 1985.

MENDES, R. C.; DIAS, D. C. F. S.; PEREIRA, M. D.; DIAS. L. A. S. Testes de vigor para avaliação do potencial fisiológico de sementes de mamona (Ricinus communis L.). Ciência e Agrotecnologia, Lavras, v. 34, n. 1, p. 114-120, 2010.

MIGUEL M. H.; CICERO, S. M. Teste de frio na avaliação do vigor de sementes de feijão. Scientia Agricola, Piracicaba, v. 56, n. 4, p. 1233-1243, 1999.

MIGUEL, M. H.; CARVALHO, M. V.; BECKERT, O. P.; MARCOS FILHO, J. Teste de frio para avaliação do potencial fisiológico de sementes de algodão. Scientia Agricola, Piracicaba, v. 58, n. 4, p. 741-746, 2001.

NAKAGAWA, J. Testes de vigor baseados no desempenho das plântulas. In: KRZYZANOWSKI, F. C.; VIEIRA, R. D.; FRANÇA-NETO, J. B. (Ed.). Vigor de sementes: conceitos e testes. Londrina: ABRATES, Cap. 2, p. 1-21, 1999.

OLIVEIRA, G. L. Testes para avaliação da qualidade fisiológica de sementes de Pinhão Manso (Jatropha curcas L.). Viçosa, 2009. 60p. Dissertação (M.S.)-Universidade Federal de Viçosa.

PEREIRA, M. D.; MARTINS FILHO, S.; LAVIOLA, B. G. Envelhecimento acelerado de sementes de pinhãomanso. Pesquisa Agropecuária Tropical, Goiânia, v. 42, n. 1, p. 119-123, 2012.

http://dx.doi.org/10.1590/S1983-40632012000100017

PINTO, T. L. F.; FILHO, J.; FORTI, V. A.; CARVALHO, C.; GOMES JUNIOR, F. G. Avaliação da viabilidade de sementes de pinhão manso pelos testes de tetrazólio e de raios X. Revista Brasileira de Sementes, Londrina, v. 31, n. 2, p. 195-2001, 2009.

SCHUAB, S. R. P.; BRACCINI, A. L.; FRANÇA-NETO, J. B.; SCAPIM, C. A.; MESCHEDE, D. K. Potencial fisiológico de sementes de soja e sua relação com a emergência das plântulas em campo. Acta Scientia Agronômica, Maringá, v. 28, n. 4, p. 553-561, 2006.

TORRES, S. B. Comparação entre testes de vigor para avaliar a qualidade fisiológica de sementes de algodão. Revista Brasileira de Sementes, Londrina, v. 20, n. 2, p. 11-15, 1998.

VANZOLINI, S.; MEORIN, E. B. K.; SILVA R. A., NAKAGAWA, J. Qualidade sanitária e germinação de sementes de pinhão-manso. Revista Brasileira de Sementes, Londrina, v. 32, n. 4, p. 009-014, 2010.

VIEIRA B. G. T. L., VIEIRA, R. D.; KRZYZANOWSKI, F. C.; FRANÇA NETO, J. B. Alternative procedure for the cold test for soybean seeds. Scientia Agricola, Piracicaba, v. 67, n. 5, p. 540-545, 2010. 